\title{
Tromboembolia pulmonar asociada al síndrome de la vena cava superior de origen trombótico
}

\section{Ana Madeleine Barrera-López*, Luis Arcadio Cortés-P. y Erika María Salazar-C.}

Departamento de Urgencias, Fundación Cardioinfantil, Instituto de Cardiología, Bogotá, Colombia

Recibido el 27 de mayo de 2016; aceptado el 4 de agosto de 2016

Disponible en Internet el 7 de noviembre de 2016

\section{PALABRAS CLAVE}

Trombo embolismo

pulmonar;

Síndrome de la vena

cava superior;

Trombosis de la vena

cava superior;

Poliglobulia o

eritrocitosis

\section{KEYWORDS}

Pulmonary

thromboembolism;

Superior vena cava

syndrome;

Superior vena cava

thrombosis;

Polyglobulia or

erythrocytosis
Resumen Se describe el caso de un paciente masculino de 32 años de edad, quien consulta al servicio de urgencias con historia clínica del síndrome de la vena cava superior, en el estudio de la angiotomografía pulmonar, se demuestra embolismo pulmonar izquierdo, trombosis de la vena yugular interna derecha y confluente yugulo subclavio. No hay evidencia de asociación con neoplasia, infecciones o enfermedades del tejido conectivo, siendo el único factor de riesgo asociado para la trombosis, hiperviscocidad (tríada de virchow) por poliglobulia.

(c) 2016 Sociedad Colombiana de Cardiología y Cirugía Cardiovascular. Publicado por Elsevier España, S.L.U. Este es un artículo Open Access bajo la licencia CC BY-NC-ND (http:// creativecommons.org/licenses/by-nc-nd/4.0/).

Pulmonary thromboembolism associated to superior vena cava syndrome thrombotic origin

Abstract The case of a 32-year old male patient who consulted the ER with a clinical record of superior vena cava syndrome is described. CT pulmonary angiography study reveals left pulmonary embolism, thrombosis in right-sided internal jugular vein and subclavian-jugular confluence. There is no evidence of association with neoplasms, infections or connective tissue disease, hyperviscosity (Virchow's triad) due to polyglobulia being the only risk associated to thrombosis.

(C) 2016 Sociedad Colombiana de Cardiología y Cirugía Cardiovascular. Published by Elsevier España, S.L.U. This is an open access article under the CC BY-NC-ND license (http:// creativecommons.org/licenses/by-nc-nd/4.0/).

\footnotetext{
* Autor para correspondencia.

Correo electrónico: abarreral@cardioinfantil.org (A.M. Barrera-López).
}

\section{Introducción}

El síndrome de la vena cava superior se caracteriza por la obstrucción parcial o completa de la vena cava superior ${ }^{1}$, de etiología principalmente neoplásica, no obstante, en 
los últimos años se aprecia un aumento progresivo de las causas benignas en relación con el uso de los dispositivos vasculares ${ }^{2}$

Para nuestro conocimiento, no existen grandes asociaciones con los eventos trombóticos sin estar asociado a la neoplasia y adicionalmente, que sean tan extensos, como es el caso de la presentación asociada la tromboembolia pulmonar, limitando esta presentación asociada a pocos reportes de $\operatorname{casos}^{3}$.

\section{Presentación del caso}

Un paciente masculino de 32 años de edad, sin antecedentes clínicos de importancia, consulta al departamento de urgencias por una sensación de masa en el cuello de 10 días de evolución, con crecimiento progresivo sin limitar la deglución, asociada a rubor facial, edema palpebral y disnea de pequeños esfuerzos. En la revisión por sistemas, no hay datos adicionales. Es un paciente obeso, somnoliento, bradipsíquico, con taquipnea, uso de músculos intercostales, desaturación al ambiente (pulsoximetría de 87\%), pletórico, con inyección conjuntival, edema palpebral y en los labios. Se evidencia en el cuello anterior la presencia de aumento del diámetro en la zona 3 , de consistencia dura no adherida a planos profundos, no doloroso, no se palpan adenopatías ni soplo carotídeo.

Inicialmente, se considera que cursaba con angioedema por lo que se inicia manejo con antihistamínico y corticoide, oxígeno suplementario, con lo cual se obtiene mejoría parcial, por lo que se establece como diagnóstico diferencial del síndrome de la vena cava superior. Entre los hallazgos positivos se encontró hipercapnia $\left(\mathrm{PcO}^{1} 37,4 \mathrm{~mm} \mathrm{Hg}\right)$, gradiente alvéolo arterial aumentado $(50,7)$, poliglobulia $\left(\mathrm{Hb}^{2}\right.$ 20,6, $\mathrm{HCto}^{3} 65 \%$ ) y $\mathrm{LDH}^{4}$ elevada (460). En la tomografía del cuello se descartan masas, se complementa el estudio con la angiotomografía del tórax, la cual evidencia un gran trombo oclusivo que compromete la vena cava superior, con extensión a su desembocadura en la aurícula derecha, tromboembolia pulmonar con compromiso segmentario en el lóbulo inferior izquierdo asociado a consolidaciones parenquimatosas, probablemente, por infartos pulmonares, probable hipertensión pulmonar precapilar y dilatación tanto auricular como ventricular derecha (figs. 1 y 2 ).

El ecocardiograma transtorácico muestra severa dilatación de las cavidades derechas, con función sistólica moderadamente comprometida y signos indirectos de hipertensión pulmonar, los marcadores de injuria del miocardio evidencian péptido natriurético cerebral de $109 \mathrm{pg} / \mathrm{ml}$, troponina I de alta sensibilidad de $0,050 \mathrm{ng} / \mathrm{ml}$ (ligeramente positiva). El doppler venoso de los miembros superiores evidencia trombosis venosa profunda de la vena yugular interna derecha y confluente yugulo subclavio sin recanalizar. Se solicita gammagrafía $v / Q^{5}$ que confirma la tromboembolia pulmonar izquierdo crónico.

\footnotetext{
1 Presión de dióxido de carbono.

2 Hemoglobina.

3 Hematocrito.

${ }^{4}$ Lactato deshidrogenasa.

5 Ventilación perfusión.
}

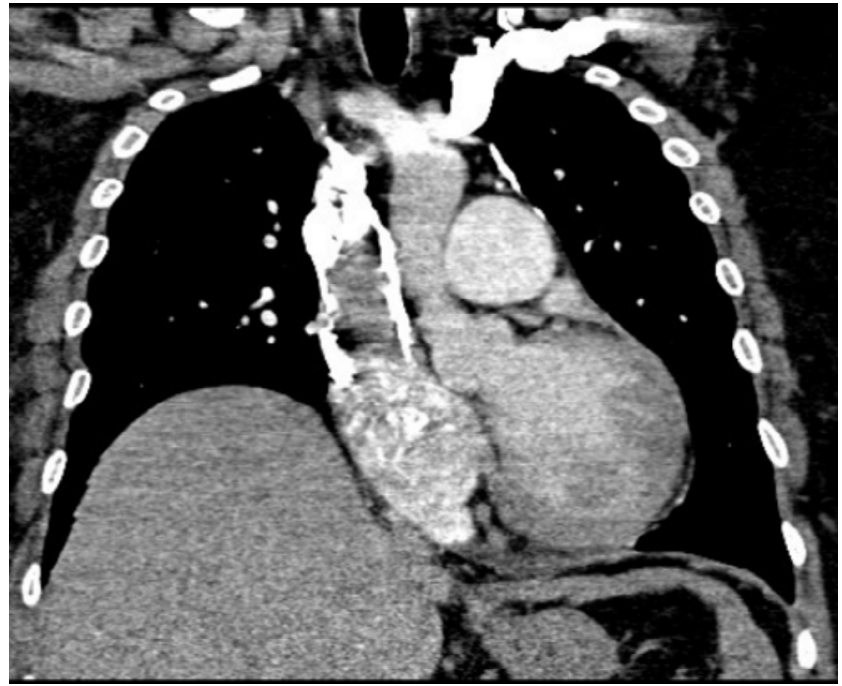

Figura 1 Trombo oclusivo hipodenso que compromete la vena cava superior, la cual se encuentra dilatada, que se extiende hasta su desembocadura en la aurícula derecha, el medio de contraste rodea el trombo.

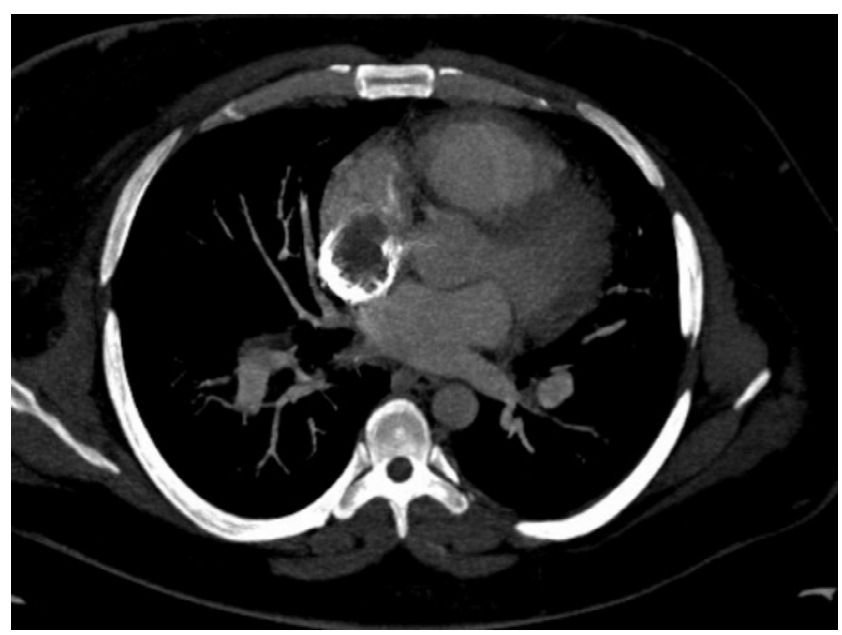

Figura 2 Signos de la tromboembolia pulmonar con oclusión segmentaria en el lóbulo inferior izquierdo, para los segmentos lateral y posterior.

Durante la estancia en el hospital, se inicia manejo anticoagulante con la heparina de bajo peso molecular y la warfarina, se realizan los estudios iniciales de trombofilia que resultan negativos (ANA, IgG y $M$ cardiolipinas) $^{6}$, aún pendientes los jak 2 y la resistencia a la proteína C, y homocisteína, alta probabilidad para el síndrome de apnea/hipopnea a estudio (Epworth 22 - escala de Berlín alto riesgo), se da egreso para continuar manejo y estudio ambulatorio.

\footnotetext{
${ }^{6}$ ANA: Anticuerpo antinuclear; IgG y M: Inmunoglobulina $G$ y $M$ : Cardiolipinas.
} 


\section{Discusión}

El médico escocés William Hunter, describió por primera vez el síndrome de la vena cava superior en el año 1957 en un paciente con aneurisma aórtico sifilítico ${ }^{1}$, tiempo en que las infecciones (histoplasmosis, tuberculosis, aspergilosis, blastomicosis, nocardiosis) eran la primera causa de obstrucción de la vena cava superior. Posteriormente, las enfermedades neoplásicas, especialmente las pulmonares, alcanzaron el $90 \%$ de las causas principales ${ }^{4}$, no obstante, en los últimos años, se han incrementado las causas benignas en relación a los eventos trombóticos por los dispositivos vasculares ${ }^{2}$, entre otras causas benignas, las enfermedades del tejido conectivo y la policitemia.

Características clínicas como el enrojecimiento y el edema facial, con edema periorbital o estridor, hacen difícil distinguir entre el síndrome de la vena cava superior con factores de riesgo desconocidos o un cuadro de angioedema idiopático o anafilaxia ${ }^{5}$, como ocurre en nuestro paciente.

La presentación clínica del síndrome de la vena cava superior, está en relación a la obstrucción del flujo de la vena cava superior y el subsecuente desarrollo de las colaterales en la pared del tórax, presentándose edema facial en el cuello y los miembros superiores, disnea, tos, síncope entre otros 6 , y su asociación con el embolismo pulmonar no es muy frecuente $\mathrm{e}^{7-10}$, siendo el diagnóstico en algunos casos hecho por la autopsia ${ }^{11-13}$.

Aunque el diagnóstico del síndrome de la vena cava superior es clínico, la evaluación inicial de todo paciente debe incluir una radiografía de tórax, en nuestro paciente era normal, siendo así en hasta el $16 \%$ de los $\operatorname{casos}^{14}$. La tomografía computarizada del tórax, ofrece mayor información diagnóstica, dado que puede determinar si una obstrucción se debe a la compresión externa o debido a la trombosis ${ }^{7}$.

Como complicaciones se han descrito: el edema laríngeo, el edema cerebral y el bajo gasto cardiaco $^{1}$, casos en los cuales el tratamiento es una emergencia e incluye los corticoides y los diuréticos, aunque su eficacia es cuestionable.

Nuestro paciente no presentaba síntomas B u otras características clínicas que sugirieran enfermedad neoplásica concurrente, tampoco los estudios imagenológicos adicionales. Sin embargo, estaba poliglobúlico, lo cual se ha descrito como factor de riesgo para la trombosis por favorecer la estasis vascular. El estudio de la poliglobulia, se debe realizar cuando encontramos un hematocrito superior al $48 \%$ en las mujeres o del $51 \%$ en los varones, en un mínimo de 2 determinaciones separadas. La causa más frecuente de la misma es la hipoxia secundaria a las enfermedades cardiopulmonares o las enfermedades mieloproliferativas como la policitemia vera, por tanto, hay que buscar los síntomas y los signos de dichas afecciones y dirigir de esta forma los estudios adicionales.

En nuestro paciente, no es claro cuál fue la causa del síndrome de la vena cava superior asociado al embolismo pulmonar, no obstante, consideramos que nuestro paciente, cursa con embolismo pulmonar crónico por la gammagrafía $\checkmark / Q$ positiva, en relación a la hiperviscosidad (tríada de virchow) por la poliglobulia, que está asociada al síndrome de apnea del sueño sin manejo, que condicionó la hipoventilación crónica. Si bien, como se ha mencionado, la poliglobulia puede ser un factor de riesgo, no encontramos reportes de casos al respecto.

Considerando que las patologías neoplásicas pueden debutar inicialmente con los fenómenos trombóticos, no se descarta que posteriormente, se documente alguna entidad oncológica.

\section{Conclusión}

Las causas no oncológicas del síndrome de la vena cava superior continúan en aumento, incluyendo las asociaciones con la trombosis extensa de miembros superiores y yugulares que pueden desencadenar incluso la tromboembolia pulmonar.

Descripciones adicionales son necesarias con el fin de establecer las predicciones de la tromboembolia pulmonar asociada síndrome de la vena cava superior para aumentar el diagnóstico premortem de este síndrome.

\section{Responsabilidades éticas}

Protección de personas y animales. Los autores declaran que para esta investigación no se han realizado experimentos en seres humanos ni en animales.

Confidencialidad de los datos. Los autores declaran que han seguido los protocolos de su centro de trabajo sobre la publicación de datos de pacientes.

Derecho a la privacidad y consentimiento informado. Los autores declaran que en este artículo no aparecen datos de pacientes.

\section{Financiación}

Apoyo financiero no requerido.

\section{Conflicto de intereses}

Los autores declaran no tener ningún conflicto de intereses.

\section{Bibliografía}

1. Nickloes TA. Superior Vena Cava Syndrome: Background, Pathophysiology, Etiology, Medscape. Updated: Sep 28, 2015.

2. Cohen R, Mena D, Carbajal-Mendoza R, Matos N, Karki N. Superior vena cava syndrome: A medical emergency? Int. J. Angiol. 2008;17:43-6.

3. Todd R. Otten, Paul D. Stein, FCCP; Kalpesh C. Patel, Syed Mustafa, and Allen Silbergleit. Thromboembolic Disease Involving the Superior Vena Cava and Brachiocephalic Veins. Chest. 2003; 123:809-812.

4. BMJ Best Practice, Superior vena cava syndrome. Updated: Jun 18, 2015.

5. Khalili B, Boggs PB, Bahna SL, Marsala AJ, Tapolyai M, Bryn RO. Superior vena cava syndrome: A masquerader of anaphylaxis. J. Allergy Clin Immunol. 2007;119 Supple 1:S29 (Abst).

6. Rice TW, Rodriguez RM, Light RW. The superior vena cava syndrome: clinical characteristics and evolving etiology. Medicine (Baltimore). 2006;85:37-42. 
7. Kwong T, Leonidas JC, Ilowite NT. Asymptomatic superior vena cava thrombosis and pulmonary embolism in an adolescent with SLE and antiphospholipid antibodies. Clin. Exp. Rheum. 1994;12:215-7.

8. Goldstein MF, Nestico P, Olshan AR, Schwartz AB. Superior vena cava thrombosis and pulmonary embolus: association with right atrial mural thrombus. Arch. Intern. Med. 1982;142:1726-8.

9. Kajiya T, Anan R, Kameko M, Mizukami N, Minagoe S, Hamasaki S, et al. Intracardiac thrombus, superior vena cava syndrome, and pulmonary embolism in a patient with Beheçt's disease: a case report and literature review. Heart Vessels. 2007;22:278-83.

10. Vivek G, Devasia T, Dias LS. Catastrophic superior vena caval thrombus with massive pulmonary thromboembolism. BMJ Case Reports. 2011, http://dx.doi.org/10.1136/bcr.02.2011.3901
11. Shah-Mirany J, Mirhoseini M, Head LR. Fatal pulmonary embolism from jugular veins following benign superior vena cava syndrome. Ann Thorac Surg. 1971;11:238-42.

12. Maddox A-M, Valdivieso M, Lukeman J, Smith TL, Barkley HE, Samuels ML, et al. Superior vena cava obstruction in small cell bronchogenic carcinoma: clinical parameters and survival. Cáncer. 1983;52:2165-72.

13. Ryan JA, Abel RM, Abbott WM, Hopkins CC, Chesney TM, Cothey $\mathrm{R}$, et al. Catheter complications in total parenteral nutrition: a prospective study of 200 consecutive patients. N Engl J Med. 1974;290:757-61.

14. Parish JM, Marschke RF Jr, Dines DE, Lee RE. Etiologic considerations in superior vena cava syndrome. Mayo Clin. Proc. 1981;56:407-13. 